\title{
Increasing lentiviral vector titer using inhibitors of protein kinase $\mathbf{R}$
}

\author{
Gilles Pernod ${ }^{1,2}$, Richard Fish ${ }^{1}$, Jia Wei Liu ${ }^{1}$, and Egbert K.O. Kruithof ${ }^{1}$ \\ ${ }^{1}$ University Hospital of Geneva, Geneva, Switzerland and ${ }^{2} \mathrm{CHU}$ Grenoble, \\ Grenoble, France \\ BioTechniques 36:576-580 (April 2004)
}

Lentiviral vectors are increasingly used as vehicles for stable gene transfer ex vivo and in vivo (1). These gene transfer vectors have a large cloning capacity, stably integrate the transgene into the host cell genome, and can transduce nondividing cells such as hematopoietic progenitor cells (2). However, in comparison with the production of retroviral or adenoviral vectors, current methods for producing lentiviral vectors are inefficient, labor-intensive, and time- and reagent-consuming. The mechanisms that limit lentiviral vector production are poorly understood. One limiting factor may be the presence of double-stranded RNA (dsRNA) generated during lentiviral vector production or after transduction of producer cells by released lentiviral vector particles. Indeed, infection of cells by viruses often results in the accumulation of dsRNA in the cytoplasm, which then activates interferon-induced antiviral defense pathways (3). Upon binding to dsRNA, the interferon-inducible protein kinase (PKR) becomes activated. Activated PKR phosphorylates the eukaryotic initiation factor- $2 \alpha$ (eIF-2 $\alpha$ ), resulting in a block in protein synthesis initiation. This leads to the inhibition of viral RNA translation, which affects the stability and processing of lentivirus-specific proteins (4-6). A second group of interferon-inducible proteins, the oligoadenylate synthetases (OAS), bind dsRNA and activate RNase L, which degrades both endogenous and viral RNA. Viruses have developed a variety of approaches to repress the innate mammalian antiviral cellular responses. One example is the adenovirus-encoded virus-associated RNA I (VAI RNA), which is transcribed by RNA polymerase III after virus infec- tion. VAI RNA binds to PKR and prevents its activation by dsRNA (7).

In the present study, we investigated whether lentiviral vector production could be increased by the inhibition of PKR activity. Human immunodeficiency virus (HIV)-based lentiviral vectors were produced by the cotransfection of human embryonic kidney (HEK) 293T cells with three plasmids, using the calcium-phosphate precipitation method (2). pCMVR8.91 (15 $\mu \mathrm{g})$ encodes the necessary HIV structural genes, pMD. $\mathrm{G}(5 \mu \mathrm{g})$ bears the vesicular stomatitis virus glycoprotein $\mathrm{G}$ (VSV-G) envelope cDNA, and pLoxCWGFP $(20 \mu \mathrm{g})$ is the transfer vector carrying the enhanced green fluorescent protein (EGFP) gene under the control of the cytomegalovirus (CMV) immediate early enhancer/ promoter region between modified HIV long terminal repeats (LTRs) (the three plasmids were generously provided by Dr. D. Trono, University Medical Center of Geneva, Geneva, Switzerland). Sixteen hours after transfection, the cell medium was replaced with $15 \mathrm{~mL}$ of fresh medium and incubated for an additional $24 \mathrm{~h}$. The conditioned medium was collected and stored at $-20^{\circ} \mathrm{C}$. Lentiviral vector titers were determined by flow cytometry analysis of EGFP expression in transduced HeLa cells. Vector titers are expressed as transduction units per milliliter $(\mathrm{TU} / \mathrm{mL})$.

We employed two approaches to study whether inhibitors of PKR increase lentiviral vector yields. In the first approach, we cotransfected the three lentiviral vector production plasmids with different amounts of the pAdVAntage ${ }^{\mathrm{TM}}$ plasmid (Promega, Madison, WI, USA) that encodes VAI RNA, an inhibitor of PKR activation, as well as VAII RNA. In the second ap- proach, we added different concentrations of 2-aminopurine (2-AP) (Sigma, St. Louis, MO, USA), an inhibitor of PKR phosphorylation (8). This inhibitor has been previously shown to increase the yield of different viruses grown on interferon-treated cells (9).

We observed that cotransfection with the pAdVAntage plasmid increased lentiviral vector titers in a dose-dependent manner (Figure 1, A and B). A maximal, two-fold increase was obtained when $10 \mu \mathrm{g}$ of pAdVAntage plasmid were used. Mean fluorescence intensities in transduced cells were not modified by cotransfection with the pAdVAntage plasmid. This indicates that the presence of pAdVAntage increased the number of lentiviral vector particles but had no effect on the transgene expression level in the transduced cells (Figure 1B). Using 2-AP, we obtained a dose-dependent increase in lentiviral vector production. Compared to controls, almost 4-fold higher titers were obtained using $5 \mathrm{mM}$ 2-AP (Figure 1, C and D). To determine the effect of PKR inhibitors on PKR activity under our experimental conditions, we analyzed the phosphorylation state of eIF- $2 \alpha$. We observed that cotransfection of 293T cells with the pAdVAntage plasmid or incubation with 2-AP markedly decreased the levels of phosphorylated eIF-2 $\alpha$ (Figure 1E). To assess activation of the OAS-mediated interferon response pathway, which ultimately results in RNase L activation, we attempted to compare the levels of OAS1 mRNA in lentiviral vector producing 293T cells with and without pAdVAntage and 2-AP. We were unable to detect OAS1 mRNA in any lentiviral vector-producing $293 \mathrm{~T}$ cells, even after 50 PCR cycles. In other cell types, OAS1 was readily detectable at PCR cycle 25 (data not shown). Therefore, limitations on lentiviral vector production are not linked to the induction of OAS1 in 293T cells. Our results show that inhibitors of PKR activity can be used to increase lentiviral vector yields in HEK 293T producer cells.

\section{ACKNOWLEDGMENTS}

This work was supported by a grant from the Association pour la Recherche 
A
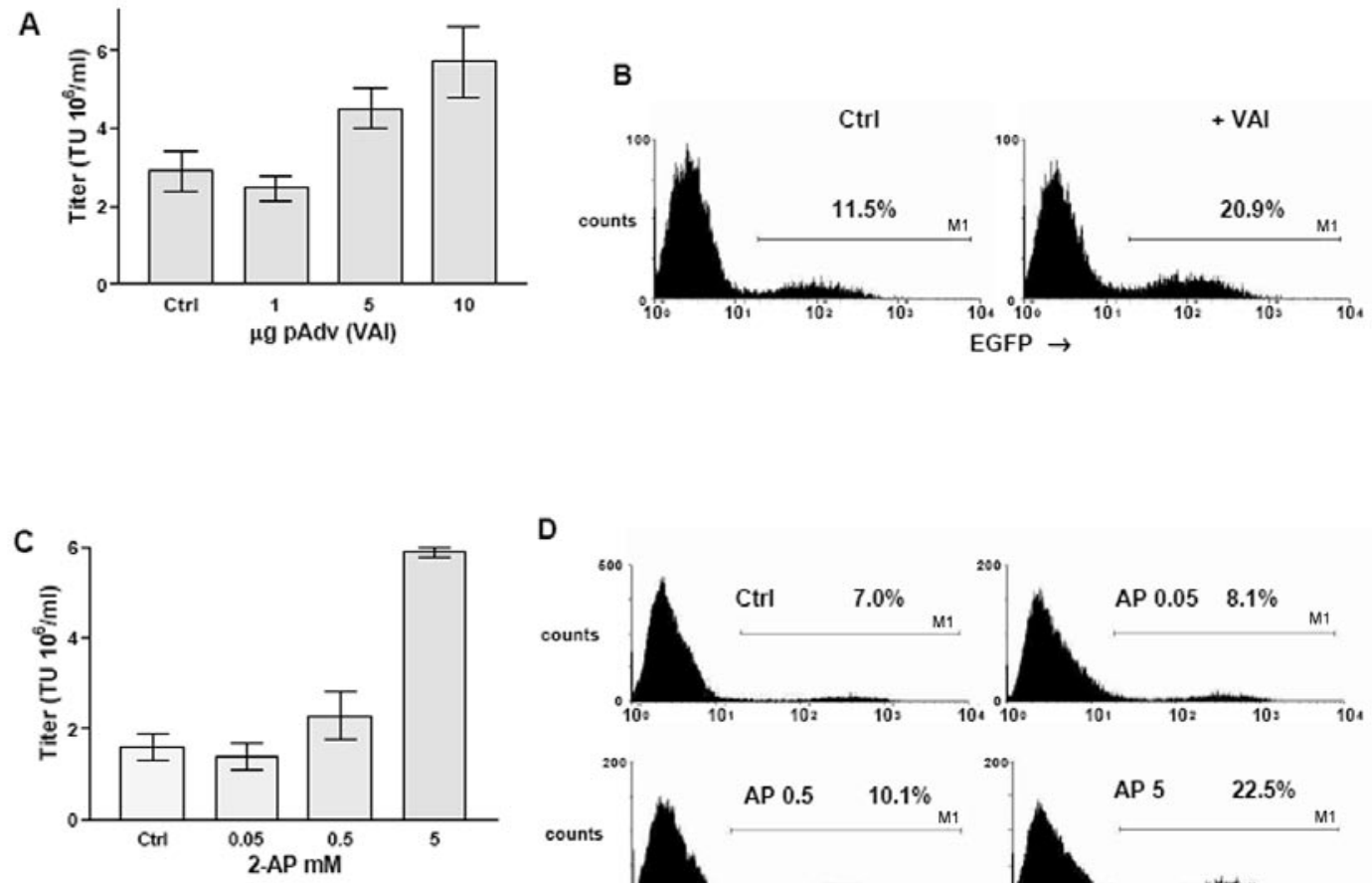

D
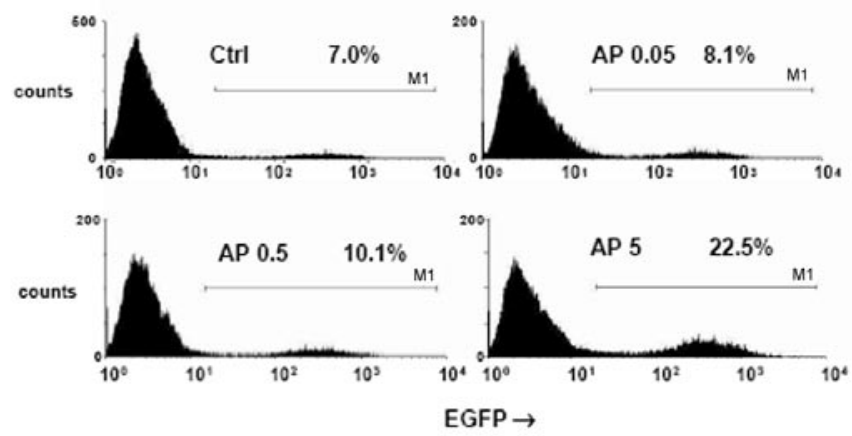

E

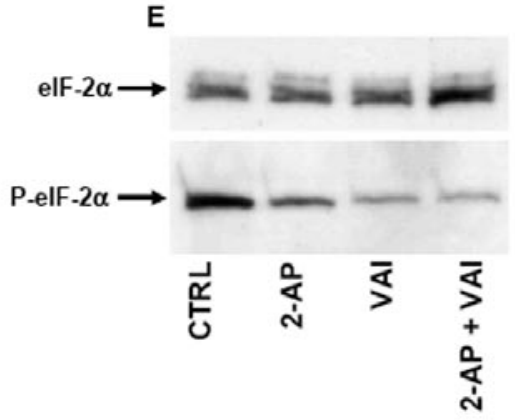

Figure 1. Protein kinase (PKR) inhibitors increase lentiviral vector titers. Lentiviral vectors were produced by cotransfection of three plasmids, as described in the text, with or without pAdVAntage or 2-aminopurine (2-AP). Briefly, 5 million human embryonic kidney (HEK) 293T cells were seeded into 100 $\mathrm{mm}$ cell culture dishes 1 day prior to transfection in $10 \mathrm{~mL}$ of Dulbecco's modified Eagle's medium (DMEM) supplemented with $10 \%$ fetal calf serum (FCS). The medium was changed at least $1 \mathrm{~h}$ prior to transfection. After transfection $(24 \mathrm{~h})$, the medium was replaced (15 mL), and vector-containing conditioned medium was collected after an additional $24 \mathrm{~h}$. Vector titers were determined on HeLa cells previously seeded in 6-well plates at 50,000 cells/well 1 day prior to transduction. The vectors were incubated with HeLa cells in medium containing $8 \mu \mathrm{g} / \mathrm{mL}$ hexadimethrine bromide (Sigma). Flow cytometry was performed 4 days after transduction. (A and B) Effect of pAdVAntage, a plasmid that encodes the adenovirus virus-associated RNA I (VAI RNA), an inhibitor of PKR activation. (A) Increasing amounts of pAdVAntage were included in transfection mixtures, and resulting vector titers were determined from the percentage of enhanced green fluorescent protein $\left(\mathrm{EGFP}^{+}\right)$transduced HeLa cells. The figure shows the mean $( \pm$SEM) of four independent experiments. (B) Representative flow cytometry analysis of HeLa cells transduced with $5 \mu \mathrm{L}$ lentiviral vectors produced without (Ctrl) or with $10 \mu \mathrm{g}$ of pAdVAntage (VAI). Percentage of EGFP ${ }^{+}$cells was determined from the M1 gating shown. (C and D) Effect of 2-AP. (C) 2-AP was included in the 293T cell medium during lentiviral vector production at the concentrations shown. Vector titers were determined as described above. The figure shows the mean ( \pm SEM) of four independent experiments. (D) Representative flow cytometric analysis of HeLa cells transduced with $5 \mu \mathrm{L}$ lentiviral vectors made without (Ctrl) or with $0.05,0.5$, or $5 \mathrm{mM} 2-\mathrm{AP}$. Percentage of $\mathrm{EGFP}^{+}$cells was determined from the M1 gating shown. (E) PKR inhibitors reduce eukaryotic initiation factor-2 $\alpha$ (eIF-2 2 ) phosphorylation in lentiviral vector-producing $293 \mathrm{~T}$ cells. Vector production was performed in control cells, in cells incubated with $5 \mathrm{mM} 2$-AP, $10 \mu \mathrm{g}$ of pAdVAntage, or $5 \mathrm{mM} 2-\mathrm{AP}$ and $10 \mu \mathrm{g}$ pAdVAntage. After vector collection, $48 \mathrm{~h}$ posttransfection, 293T vector-producing cells were washed in phosphate-buffered saline (PBS) and lysed in phosphorylation lysis buffer $\left(20 \mathrm{mM}\right.$ Tris- $\mathrm{HCl}, \mathrm{pH} 7.4,150 \mathrm{mM} \mathrm{NaCl}, 10 \mathrm{mM}$ EDTA, $\left.1 \mathrm{mM} \mathrm{Zn}\left(\mathrm{C}_{2} \mathrm{H}_{3} \mathrm{O}_{2}\right)_{2}, 1 \mathrm{mM} \mathrm{NaF}, 1 \mathrm{mM} \mathrm{Na} \mathrm{VO}_{4}\right)$ supplemented with Complete Mini Protease Inhibitor Cocktail (Roche, Basel, Switzerland). Cell lysates were cleared by centrifugation at $16,000 \times g$ for 5 min, and protein content was measured using the Bio-Rad ${ }^{\circledR}$ Protein Assay (Bio-Rad Laboratories, Hercules, CA, USA), with bovine serum albumin (BSA) as a standard. Twenty-eight micrograms of total protein were separated by sodium dodecyl sulfate-polyacrylamide gel electrophoresis (SDS-PAGE) and transferred to nitrocellulose membranes for immunoblotting with either anti-eIF- $2 \alpha$ or antiphosphorylated eIF-2 $\alpha$ antibodies (both from Cell Signaling Technologies, Beverly, MA, USA). Specific bands were detected using goat anti-rabbit-horseradish peroxidase (HRP; Bio-Rad Laboratories) secondary antibodies and ECL ${ }^{\circledR}$ Western Blotting Detection Reagents (Amersham Biosciences, Uppsala, Sweden). TU/mL, transduction unit per milliliter. 
contre le Cancer (G.P.), by the Groupement des Entreprises Françaises dans la Lutte contre le Cancer (GEFLUC) (G.P.), by the Swiss Foundation for Cardiology (E.K.O.K.), by the Swiss Cancer League (E.K.O.K.), by the Cancer League of Geneva (E.K.O.K.), and by the Swiss National Fund (grant no. 3200-061510.00) (E.K.O.K.).

\section{REFERENCES}

1.Trono, D. 2000. Lentiviral vectors: turning a deadly foe into a therapeutic agent. Gene Ther. 11:20-23.

2.Salmon, P., V. Kindler, O. Ducrey, B. Chapuis, R.H. Zubler, and D. Trono. 2000. High-level transgene expression in human hematopoietic progenitors and differentiated blood lineages after transduction with improved lentiviral vectors. Blood 96:33923398.

3.Katze, M.G. and M. Gale. 1997. What happens inside lentivirus or influenza virus infected cells: insights into regulation of cellular and viral protein synthesis. Methods 11:383-401

4.Clemens, M.J. and A. Elia. 1997. The double-stranded RNA-dependant protein kinase PKR: structure and function. J. Interferon $\mathrm{Cy}$ tokine Res. 17:503-524.

5.Samuel, C.E. 2001. Antiviral actions of interferons. Clin. Microbiol. Rev. 14:778-809.

6.Agy, M.B., R.L. Acker, C.H. Sherbert, and M.G. Katze. 1995. Interferon treatment inhibits virus replication in HIV-1 and SIV-infected CD4+ T-cell lines by distinct mechanisms: evidence for decreased stability and aberrant processing of HIV-1 proteins. Virology 214:379-386.

7.O'Malley, R.P., T.M. Mariano, J. Siekierka, and M.B. Mathwes. 1986. A mechanism for the control of protein synthesis by adenovirus VARNAI. Cell 44:391-400.

8.Hu, Y. and T.W. Conway. 1993. 2-aminopurine inhibits the double-standed RNA-dependant protein kinase both in vitro and in vivo. $\mathrm{J}$. Interferon Res. 13:323-328.

9.Rowland, R.R., B. Robinson, J. Stefanick, T.S. Kim, L. Guanghua, S.R. Lawson, and D.A. Benfield. 2001. Inhibition of porcine reproductive and respiratory syndrome virus by interferon-gamma and recovery of virus replication with 2-aminopurine. Arch. Virol. 146:539-555

Received 21 October 2003; accepted 21 January 2004.

Address correspondence to Egbert K.O. Kruithof, Division of Angiology and Hemostasis, Department of Internal Medicine, University Hospital of Geneva, $\mathrm{CH}$ 1211 Geneva, Switzerland. e-mail: egbert. kruithof@hcuge.ch

\title{
Monitoring aerobic Escherichia coli growth in shaken microplates by measurement of culture fluorescence
}

\author{
Hartmut F. Zimmermann, Harald Trauthwein, Uwe Dingerdissen, Mechthild
} Rieping, and Klaus Huthmacher

Degussa AG, Frankfurt am Main, Germany

BioTechniques 36:580-584 (April 2004)

Combining the advantages of both the microplate (high-throughput, ease of automation) and the classic shaken Erlenmeyer flask (adequate mixing and oxygen supply), shaken 48-well microplates are an excellent small-scale fermentation system for microbial cultivation (1). Most physiological studies are at least semiquantitative (2), and real-time biomass estimation during a bioprocess is an important goal (3). Therefore, a simple method for the monitoring of microbial growth in these 48-well plates is necessary.

Intracellular NADH and NADPH in living microorganisms can be excited to fluorescence $(460 \mathrm{~nm})$ by irritating the cells with UV light (366 nm). This fact is used as the basis of a noninvasive online method for fermentation process monitoring in stirred tank bioreactors (3-5). Since fluorescence readers for microplates are common available analytical instruments, the adaptation of this technique to the microplate should be accomplishable. Researchers, however, have been using either turbidity measurements $(6,7)$ or specially designed microplates with integrated fluorophores (8-10) for growth monitoring in multiwell plates during highthroughput screening (HTS).

The optical density or absorbance (A) of a suspension diluted in such a way that light scattering centers are essentially independent is directly proportional to the biomass concentration (C) (2):

$$
\mathrm{A}=k \times \mathrm{C}
$$
where $k$ is the proportionality constant. Biomass $\mathrm{C}$ can be calculated from NAD $(\mathrm{P}) \mathrm{H}$-dependent fluorescence $(\mathrm{F})$ by $(4,5)$ :

$$
\mathrm{C}=[\exp (-b) \times \mathrm{F}]^{1 / a}
$$
where $a$ and $b$ are constants. Equation 1 may then be written similarly to Equation 2 as:

$$
\mathrm{A}=\left[\exp \left(-b^{*}\right) \times \mathrm{F}\right]^{1 / a}
$$
where $b^{*}=b-(a \times \ln k)$.

To verify that Equation 3 is likewise true in microscale, both the absorbance and the fluorescence of serial dilutions of two JM101 cultures grown in two different media were determined.

Terrific broth (TB) and Luria Bertani broth (LB) (both from Sigma, St. Louis, MO, USA) were used as media and inoculated with $1 \%(\mathrm{v} / \mathrm{v})$ of a JM101 glycerol stock with $5 \times 10^{8}$ viable cells $/ \mathrm{mL}$. The reaction vessels were glass Erlenmeyer flasks $(100 \mathrm{~mL}$, four baffles) and flat-bottom transparent polystyrene arrays, namely nontreated 48-well cell culture plates (BD Biosciences Discovery Labware, Bedford, MA, USA) and 96-well microplates (Greiner, Frickenhausen, Germany). Two flasks were filled with $10 \mathrm{~mL}$ inoculated $\mathrm{TB}$ and $\mathrm{LB}$, respectively, closed with a cotton plug, and incubated for $18 \mathrm{~h}$ at $37^{\circ} \mathrm{C}$ on a rotary shaker with $25 \mathrm{~mm}$ diameter at $180 \mathrm{rpm}$. Then three serial dilutions of both cultures in respective media were pipeted, each in both a 48 -well plate $(400 \mu \mathrm{L} /$ well $)$ and a 96-well plate $(200 \mu \mathrm{L} /$ well $)$ using a Beckman 2000 liquid handler (Beckman, Fullerton, CA, USA). Absorbance was determined by reading the 96 -well plate in a SpectraMax ${ }^{\circledR}$ reader (Molecular Devices, Sunnyvale, CA, USA) at $620 \mathrm{~nm}$. Pure media were used as blanks, and data was converted to $1 \mathrm{~cm}$ path length. A linear correlation is only true if $\mathrm{A}<0.5$ (7). Higher $\mathrm{A}$ values, however, may be calculated from more diluted samples. The means of each 3fold measurement, as well as the results from calculations, are shown in Figure $1 \mathrm{~A}$. $\mathrm{F}$ was determined by reading the 48-well plate in a FLUOstar reader (BMG, Offenburg, Germany) using the following crucial settings: no. of flash- 